\title{
Certolizumab Pegol erhielt EMA-Zulassung
}

Die Europäische Arzneimittel-Agentur (EMA) genehmigte eine Indikationserweiterung für Certolizumab Pegol (CIMZIA ${ }^{\oplus}$ ). Diese schließt nun erwachsene Patienten mit mittelschwerer bis schwerer Plaque-Psoriasis ein [1]. Mit dieser Zulassung ist Certolizumab Pegol das erste Fc-freie, PEGylierte Anti-TNF mit einer Behandlungsoption in dieser Indikation.

"Trotz der verfügbaren Behandlungsoptionen erfahren viele Patienten mit mittelschwerer bis schwerer PlaquePsoriasis noch immer eine unzureichende Kontrolle der Krankheitssymptome, was ihre Lebensqualität erheblich beeinträchtigen kann. Diese Zulassung stellt Patienten und Ärzten ein Biologikum zur Verfügung, das zehn Jahre klinische Erfahrung bei zahlreichen entzündlichen Erkrankungen aufweist. Psoriasis-Patienten in Europa könnte Certolizumab Pegol somit eine dauerhafte Krankheitskontrolle ermöglichen", so Prof. Dr. Michael Sticherling, Leitender Oberarzt und stellvertretender Klinikdirektor der Hautklinik der Universitätsklinik Erlangen.

\section{Die Studien CIMPASI-1, CIMPASI-2 und CIMPACT}

Die Zulassung der EMA basiert auf den Daten eines klinischen Phase 3 Programms, das die Studien CIMPASI-1, CIMPASI-2 und CIMPACT beinhaltet [2]. Diese Studien bestätigten die Wirksamkeit und Verträglichkeit von Certolizumab Pegol bei der Behandlung von erwachsenen Patienten mit einer mittelschweren bis schweren Plaque-Psoriasis. Die klinischen Verbesserungen wurden bis Woche 48 aufrechterhalten [2].

In jeder der drei Studien wurde unter Verwendung des Psoriasis Area and Severity Index (PASI 75) der Anteil der Patienten gemessen, bei denen mindestens $75 \%$ oder eine noch höhere Krankheitsverbesserung gegenüber Baseline erzielt wurde sowie der Prozentsatz der Patienten, die mindestens eine Zwei-Punkte-Verbesserung auf einer Fünf-Punkte-Skala des PGA Quelle. Presseaussendung der UCB Phar$\mathrm{ma} \mathrm{GmbH}$ ten, bis zu einem endgültigen Wert, der eine vollständige oder fast vollständige Abheilung der Hautsymptome im Vergleich zu Plazebo repräsentierte [2].

Laut aktualisierter Fachinformation beträgt die empfohlene Anfangsdosis von Certolizumab Pegol für erwachsene Patienten mit Plaque-Psoriasis $400 \mathrm{mg}$ in den Wochen 0, 2 und 4. Nach der Anfangsdosis von Certolizumab Pegol beträgt die Erhaltungsdosis für erwachsene Patienten mit Plaque-Psoriasis $200 \mathrm{mg}$ alle zwei Wochen (Q2 W). Bei Patienten mit unzureichendem Ansprechen kann eine Dosis von $400 \mathrm{mg}$ Q2 W erwogen werden [1].

Certolizumab Pegol ist außerdem für die Behandlung von Psoriasis Arthritis (PsA) zugelassen, einer häufigen Komorbidität bei Psoriasis-Patienten [3, 4].

\section{Psoriasis und Schwangerschaft}

Trotz der Fortschritte in der Arzneimittelentwicklung im letzten Jahrzehnt, lassen Daten aus Patientenbefragungen vermuten, dass die mittelschwere bis schwere Psoriasis immer noch nicht ausreichend behandelt wird [5].

Besonders während der Schwangerschaft kann eine Psoriasis viele Herausforderungen mit sich bringen. $23 \%$ der Frauen erfahren eine Verschlechterung der Symptome während der Schwangerschaft [6]. Mehrere Studien haben gezeigt, dass Psoriasis Auswirkungen auf den Schwangerschaftsverlauf haben kann. Darunter ein erhöhtes Risiko für eine Frühgeburt, ein niedriges Geburtsgewicht oder eine Fehlgeburt.

Zudem kann der Säugling zu groß sein gemessen am entsprechenden Reifealter bzw. ein höheres Geburtsgewicht haben gemessen am Durchschnittsgewicht [7-9]. Darüber hinaus erfahren bis zu 65 \% der Frauen mit Psoriasis eine Verschlechterung der Symptome nach der Schwangerschaft [6]. Eine Krankheitskontrolle vor, während und nach der Schwangerschaft ist für die Gesundheit von Mutter und Säugling von immenser Bedeutung [10].

\section{Literatur}

1. European Medicines Agency (EMA), 8 June 2018

2. UCB data on file

3. CIMZIA (2018) Summary of Product Characteristics (SmPC). http://www.ema.europa. eu/docs/en_GB/document_library/EPAR_Product_Information/human/001037/ WC500069763.pdf. Zugegriffen: 23 Mai 2018

4. Oliveira Rocha B et al (2015) Psoriasis: classical and emerging comorbidities. An Bras Dermatol 90(1):9-20

5. Lebwohl MG, Bachelez H, Barker J, et al (2014) Patient perspectives in the management of psoriasis: results from the population-based Multinational Assessment of Psoriasis and Psoriatic Arthritis Survey. J Am Acad Dermatol 70(5):871-881

6. Murase JE, Chan KK, Garite TJ, et al (2005) Hormonal effect on psoriasis in pregnancy and post partum. Arch Dermatol 141(5):601-606

7. Amiri $\mathrm{N}$ et al (2016) Pregnancy comorbibidities and outcomes in psoriasis and psoriatic arthritis: a prospective cohort study. Arthritis Rheum 68(suppl 10). Abstract 2443; p3219

8. Bobotsis R, Gulliver WP, Monaghan K, et al (2016) Psoriasis and adverse pregnancy outcomes: a systematic review of observational studies. Br J Dermatol. 175(3):464-472

9. Lima XT, Janakiraman V, Hughes MD, et al (2012) The impact of psoriasis on pregnancy outcomes. J Invest Dermatol 132(1):85-91

10. Porter ML, Lockwood SJ, Kimball AB (2017) Update on biologic safety for patients with psoriasis during pregnancy. Int J Women's Dermatol 3(1):21-25

hautnah $2018 \cdot 17: 82$

https://doi.org/10.1007/s12326-

018-0288-2

(c) Springer-Verlag GmbH Austria, ein Teil von Springer Nature 2018 